TRANSACTIONS OF THE

AMERICAN MATHEMATICAL SOCIETY

Volume 355, Number 7, Pages 2771-2793

S 0002-9947(03)03265-3

Article electronically published on February 25, 2003

\title{
FORMALITY IN AN EQUIVARIANT SETTING
}

\author{
STEVEN LILLYWHITE
}

\begin{abstract}
We define and discuss $G$-formality for certain spaces endowed with an action by a compact Lie group. This concept is essentially formality of the Borel construction of the space in a category of commutative differential graded algebras over $R=H^{\bullet}(B G)$. These results may be applied in computing the equivariant cohomology of their loop spaces.
\end{abstract}

\section{INTRODUCTION}

In this paper we consider $G$-spaces and give formality results for them in an equivariant category. More specifically, given a $G$-space $M$, we discuss formality of the Borel construction $E G \times_{G} M$ or, equivalently, formality of the complex $A_{G}^{\bullet}(M)$ of equivariant differential forms. However, in the equivariant setting, the map $M \rightarrow\{p t$. $\}$ is replaced by $E G \times_{G} M \rightarrow B G$, and consequently all the commutative differential graded algebras involved are naturally $R$-algebras, where $R=H^{\bullet}(B G)$. Thus formality may be considered in the category of commutative differential graded $R$-algebras. We shall also consider the augmented case, corresponding to equivariant base points, which are the same thing as fixed points of the group action. We should like to call a $G$-space $M$ "equivariantly formal" when its Borel construction is formal in the above sense. However, the term "equivariant formality" has come to be used to describe the degeneration of the spectral sequence of the fibration $M \rightarrow E G \times_{G} M \rightarrow B G$, owing to the pervasive influence of [11. Thus we shall adopt the terminology " $G$-formal" in this paper.

We give some general results concerning $G$-formality of products and wedges and reductions to subgroups. This is followed by several examples of $G$-formal spaces, including compact Kähler manifolds and formal elliptic spaces, among others. Of course, we must make appropriate assumptions on the $G$-actions of these spaces for the results to hold.

As an application of these results, we compute the equivariant cohomology of loop spaces. (If $M$ is a $G$-space, then so is the loop space of $M$ in the obvious way.) Our motivation comes from considering the cohomology of symplectic quotients of loop spaces, see [18, although the results are of general topological interest. We shall use an "equivariant" bar complex to compute the equivariant cohomology of the loop space. If the $G$-space $M$ is $G$-formal, then the bar complex, which is generally a double complex, loses a differential and becomes a single complex, allowing for some easier calculations. In the last section we compute an example.

Received by the editors January 1, 2002.

2000 Mathematics Subject Classification. Primary 55P62; Secondary 55N91, 18G55, 57T30.

Key words and phrases. Rational homotopy theory, equivariant cohomology, bar complexes, loop spaces, homotopical algebra.

(C)2003 American Mathematical Society 
In an appendix, we discuss bar complexes and Eilenberg-Moore theory concerning the pull-back of a fibration. We also consider equivariant versions of these results, which are used in several of the proofs in the main body of the paper.

In what follows, we shall generally assume that $G$ is a compact, connected Lie group and that all spaces are connected. Whenever we need to use the localization theorem in equivariant cohomology, we shall assume that the spaces under consideration are of the homotopy type of finite-dimensional $G$-CW-complexes, and furthermore that they have finitely many connective orbit types, meaning that the set $\left\{\left[G_{x}^{0}\right] \mid x \in M\right\}$ is finite, where $G_{x}$ is the stabilizer subgroup at $x, G_{x}^{0}$ is the connected component of the identity, and $\left[G_{x}^{0}\right]$ denotes the set of conjugacy classes in $G$. This latter condition is automatically satisfied, by the way, if $M$ is compact or if $G=S^{1}$.

I would like to extend my appreciation to Chris Allday, who took the time to read the manuscript and offered advice on several key points. In particular, Proposition 4.7 is due to him.

\section{2. $k \mathcal{C D} \mathcal{G} \mathcal{A}$ AND FORMALITY}

In this section we recall some important facts about the category of commutative differential graded algebras, the notion of formality, and the connection with rational homotopy theory. We shall assume for now that our algebras are $k$-algebras, where $k$ is a field of characteristic zero. We shall denote by $k \mathcal{C D} \mathcal{G} \mathcal{A}^{o}$ the category of commutative differential graded $k$-algebras that are concentrated in non-negative degrees and have a differential that raises the degree by one. We assume further that $H^{0}(A) \approx k$, for all $A$ in $k \mathcal{C D} \mathcal{G} \mathcal{A}^{o}$. We shall denote by $k \mathcal{C D G} \mathcal{A}$ the category of algebras in $k \mathcal{C D G} \mathcal{A}^{o}$ that are augmented over $k$ (i.e., there exists for each $A$ a map $\varepsilon: A \rightarrow k$, with $k$ concentrated in degree zero), together with augmentationpreserving maps for morphisms. We shall call an object of $k \mathcal{C D G} \mathcal{A}\left(\right.$ resp. $k \mathcal{C D G} \mathcal{A}^{o}$ ) a $k$ CDGA (resp. $k \mathrm{CDGA}^{o}$ ).

We recall Quillen's abstract approach to homotopy theory, [22, [23]. He begins by defining the notion of a closed model category. A closed model category is a category, $\mathcal{C}$, with 3 distinguished classes of morphisms, called cofibrations, fibrations, and weak equivalences, which satisfy a number of axioms. The homotopy category, $H o \mathcal{C}$, is defined to be the localization of $\mathcal{C}$ with respect to the class of weak equivalences. Quillen introduces a notion of homotopy and shows that $H_{o} \mathcal{C}$ is equivalent to the more concrete category ho $\mathcal{C}$ which has for its objects the cofibrant/fibrant objects of $\mathcal{C}$, and for its morphisms the homotopy classes of maps. We point out the important fact that two objects $X$ and $Y$ in $H o \mathcal{C}$ are isomorphic if and only if there exists a chain (in $\mathcal{C}$ ) of weak equivalences

$$
X \leftarrow Z_{1} \rightarrow Z_{2} \leftarrow \cdots \leftarrow Z_{n} \rightarrow Y .
$$

In [4] it is shown that the categories $k \mathcal{C D G} \mathcal{A}^{o}$ and $k \mathcal{C D G} \mathcal{A}$ are closed model categories where the weak equivalences are the quasi-isomorphisms (maps that induce an isomorphism on cohomology), fibrations are the surjective morphisms, and cofibrations are maps that satisfy the following lifting condition: a map $f$ is a 
cofibration if for every commutative diagram

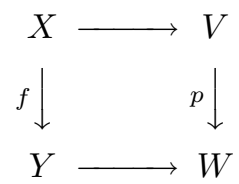

with $p$ a fibration and weak equivalence, there is a map from $Y$ to $V$ making the diagram commute. (Actually, in [4], the authors do not assume that $H^{0}(A) \approx k$ for all algebras $A$. We have included this assumption for ease of presentation, but the difference is slight.)

Given a closed model category, $\mathcal{C}$, with initial object $*$, an object $B$ is called cofibrant if the map $* \rightarrow B$ is a cofibration. $B$ is called a cofibrant model for $A$ if $B$ is cofibrant and there exists a weak equivalence $B \rightarrow A$. It follows from the axioms for a closed model category that every object in a closed model category has a cofibrant model. Moreover, there are various lifting and homotopy results associated with cofibrant algebras; see 4], section 6 . We mention one here. If $\varphi: B_{1} \rightarrow B_{2}$ is a quasi-isomorphism, and we have a map $f: A \rightarrow B_{2}$ with $A$ cofibrant, then there exists a lift $\tilde{f}: A \rightarrow B_{1}$ such that $\varphi \tilde{f} \simeq f$, where $\simeq$ denotes homotopy.

Note that $k \mathcal{C D} \mathcal{G} \mathcal{A}$ is pointed with point object $k$. The homotopy groups of a $k$ CDGA $A$ are defined to be

$$
\pi^{n} A \stackrel{\text { def }}{=} H^{n}\left(\bar{A} /(\bar{A})^{2}\right)
$$

where $\bar{A}=\operatorname{ker} \varepsilon$, for $\varepsilon: A \rightarrow k$ a given augmentation of $A$.

If $f: B_{1} \rightarrow B_{2}$ is a weak equivalence of cofibrant $k$ CDGA's, then $f_{*}: \pi^{\bullet} B_{1} \rightarrow$ $\pi^{\bullet} B_{2}$ is an isomorphism. Thus, if we define $\Pi^{n}(A) \stackrel{\text { def }}{=} \pi^{n}(B)$ for $B$ a cofibrant model of $A$, then $\Pi^{n}(A)$ is well-defined up to isomorphism. Moreover, if $f: A_{1} \rightarrow A_{2}$ is a map of $k$ CDGA's, then $f$ induces a unique homotopy class of maps $f: B_{1} \rightarrow B_{2}$, for fixed choices of cofibrant models $B_{1}, B_{2}$ of $A_{1}, A_{2}$, respectively. It follows that there is a unique map $f_{*}: \Pi\left(A_{1}\right) \rightarrow \Pi\left(A_{2}\right)$. Thus $\Pi$ is functorial, and different choices of cofibrant models yield naturally isomorphic such functors.

In $k \mathcal{C D} \mathcal{G} \mathcal{A}$, there is a special class of cofibrant models called minimal models. A minimal model of an algebra $A$ is defined to be a cofibrant model, $\mathcal{M} \rightarrow A$, that is connected $\left(\mathcal{M}^{0} \approx k\right)$, and such that the induced differential on $\overline{\mathcal{M}} /(\overline{\mathcal{M}})^{2}$ is zero. It can be shown that each algebra in $k \mathcal{C D} \mathcal{G} \mathcal{A}$ has a minimal model, unique up to isomorphism. If $M$ is a path-connected topological space, the (pseudo-dual) $k$ homotopy groups of $M$ are defined to be $\Pi^{n}(M) \stackrel{\text { def }}{=} \Pi^{n}\left(A^{\bullet}(M)\right)=\pi^{n}(\mathcal{M})$, where $\mathcal{M}$ is a minimal model for $A^{\bullet}(M)$. Here, $A^{\bullet}(M)$ denotes the Sullivan-de Rham complex, which is a $\mathbb{Q} C D G A$; see, for example, 3 for the definition. If $M$ is a smooth manifold, we may also use the ordinary de Rham complex, taking $k$ to be $\mathbb{R}$.

Halperin has explicitly identified the cofibrations (and hence cofibrant objects) in $k \mathcal{C D} \mathcal{G} \mathcal{A}$. Cofibrations are the so-called KS-extensions, and the cofibrant objects are the KS-complexes. Since these notions will be important to us, we give their definitions here; see [14] or [3].

Definition 2.1. A map $f: A \rightarrow B$ of $k$ CDGA's is said to be a $K S$-extension if there exists a well-ordered subset $E \subset B, E=\left\{x_{\alpha}\right\}$, such that $A \otimes \wedge(E) \rightarrow B$ is an isomorphism of commutative graded algebras, where $\bigwedge(E)$ denotes the free 
graded commutative algebra on the set $E$, and the map is induced by $f$ and the inclusion of $E \subset B$. Identifying $B$ with $A \otimes \wedge(E)$, the differential on $B$ satisfies

(1) $d_{B}(a \otimes 1)=d_{A}(a) \otimes 1$,

(2) $d_{B}\left(1 \otimes x_{\alpha}\right) \in A \otimes \bigwedge\left(E_{<\alpha}\right)$,

where $E_{<\alpha}=\left\{x_{\beta} \mid \beta<\alpha\right\}$. If $E$ also satisfies $\operatorname{deg}\left(x_{\alpha}\right)>0 \forall x_{\alpha} \in E$, and $\operatorname{deg}\left(x_{\beta}\right)<$ $\operatorname{deg}\left(x_{\alpha}\right) \Rightarrow \beta<\alpha$, then $f$ is called a minimal KS-extension. If $A=k$, then we replace the word "extension" by the word "complex" in the definition, obtaining the notion of $K S$-complex. (A minimal KS-complex is the same thing as a minimal algebra, defined above.) A minimal KS-extension in which $A$ is also minimal is called a $\Lambda$-minimal $\Lambda$-extension. Note that in a (minimal) KS-extension, $\Lambda(E)$ is a (minimal) KS-complex, with differential such that $\varepsilon \otimes 1: A \otimes \bigwedge(E) \rightarrow \bigwedge(E)$ is a map of $k \mathrm{CDGA}^{o}$ 's, where $\varepsilon$ is the augmentation of $A$. Moreover, all of these maps may be made compatible with augmentations.

If $A$ is a $k$ CDGA, then its cohomology, $H(A)$, may be considered to be a $k$ CDGA with zero differential.

Definition 2.2. $A$ is said to be formal if $A \approx H(A)$ in $H o(k \mathcal{C D G} \mathcal{A})$.

It is easy to see that this definition is equivalent to the following two.

Lemma 2.3. Consider the category $k \mathcal{C D} \mathcal{G} \mathcal{A}$. The following are equivalent:

(1) A is formal.

(2) There is a diagram

$$
A \leftarrow B \rightarrow H(A),
$$

where the maps are weak equivalences and $B$ is a cofibrant model for $A$. (In particular, we may pick $B$ to be minimal.)

(3) There is a chain of quasi-isomorphisms

$$
A \leftarrow A_{1} \rightarrow A_{2} \leftarrow \cdots \leftarrow A_{n} \rightarrow H(A) .
$$

This theory has an important application to rational homotopy theory. It turns out that the homotopy category of rational finite $\mathbb{Q}$-type nilpotent spaces is equivalent to the homotopy category of the full subcategory of $\mathbb{Q C} \mathcal{D G} \mathcal{A}$ consisting of algebras $A$ with $\Pi A$ of finite type, [4]. Thus we may "do" rational homotopy theory in a category of differential graded algebras. As an example, if $X$ is a path-connected, simply-connected topological space of finite $\mathbb{Q}$-type, then there is a natural isomorphism

$$
\Pi^{n}\left(A^{\bullet}(X)\right) \approx \operatorname{Hom}_{\mathbb{Q}}\left(\pi_{n}(X) \otimes \mathbb{Q}, \mathbb{Q}\right),
$$

where $A^{\bullet}(X)$ is the $\mathbb{Q}$ CDGA of Sullivan-de Rham differential forms on $X$. If $X$ is a smooth manifold, the same statement for homotopy groups holds if we use instead the de Rham algebra $A^{\bullet}(X)$ and replace $\mathbb{Q}$ coefficients by $\mathbb{R}$, or $\mathbb{C}$. There is not a corresponding equivalence of homotopy categories over $\mathbb{R}$ or $\mathbb{C}$, however.

A path-connected topological space is said to be formal if its Sullivan-de Rham algebra $A^{\bullet}(X)$ is formal. If $X$ is a smooth manifold, we may use the de Rham algebra and real or complex coefficients. However, a well-known result in rational homotopy theory states that formality over $\mathbb{R}$ or $\mathbb{C}$ implies formality over $\mathbb{Q}$; see, for example, [15]. 
Formal spaces include compact Kähler manifolds and many homogeneous spaces, including compact globally symmetric spaces. Products, wedges, and connected sums of formal spaces are again formal. The topological consequences of formality include the vanishing of all Massey products. Moreover, the rational homotopy type of such a space is determined solely by its cohomology algebra (at least for a large class of such spaces).

\section{3. $R \mathcal{C D} \mathcal{G} \mathcal{A}$ AND $G$-FORMALITY}

In this paper, we shall be concerned with equivariant versions of standard formality results. Let $G$ be a compact, connected Lie group. Then $H^{\bullet}(B G ; k)$ is isomorphic to the $k \mathrm{CDGA}$ freely generated by a finite number of generators of even degree. We shall denote $R \stackrel{\text { def }}{=} H^{\bullet}(B G)$. We define the category $R \mathcal{C D} \mathcal{G} \mathcal{A}^{o}$ to be the category of commutative differential graded $R$-algebras. We shall continue to assume that $H^{0}(A) \approx k$ for all algebras $A$. Thus, we obtain a faithful forgetful functor from $R \mathcal{C D} \mathcal{G} \mathcal{A}^{o}$ to $k \mathcal{C D} \mathcal{G} \mathcal{A}^{o}$. We also define $R \mathcal{C D G} \mathcal{A}$ to be the category of commutative differential graded $R$-algebras augmented over $R$. Composing augmentations with the augmentation $R \rightarrow k$, we get a faithful forgetful functor from $R \mathcal{C D G} \mathcal{A}$ to $k \mathcal{C D} \mathcal{G} \mathcal{A}$

It is a standard result that if $\mathcal{C}$ is a closed model category and $B$ is an object of $\mathcal{C}$, then the "over category" $\mathcal{C} / B$ whose objects are maps $X \rightarrow B$ and whose morphisms are commutative squares of the type

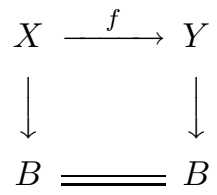

may be given the structure of a closed model category with the following definitions. Such a morphism in $\mathcal{C} / B$ will be called a fibration, cofibration, or weak equivalence, if the map $f: X \rightarrow Y$ is such in $\mathcal{C}$. A similar statement holds for the "under category", $B \backslash \mathcal{C}$. See [7] for these and other results about closed model categories.

Thus we see that both $R \mathcal{C D G} \mathcal{A}^{o}=R \backslash k \mathcal{C D} \mathcal{G} \mathcal{A}^{o}$ and $R \mathcal{C D G} \mathcal{A}=R \mathcal{C D} \mathcal{G} \mathcal{A}^{o} / R$ are closed model categories. Moreover, the simplicial category structure on $k \mathcal{C D} \mathcal{G} \mathcal{A}^{o}$ defined in [4], section 5, induces a simplicial category structure on $R \mathcal{C D} \mathcal{G} \mathcal{A}^{\circ}$ and $R \mathcal{C D G} \mathcal{A}$ in such a way that the results of 4, section 5, suitably modified, hold for these categories as well (cf. 22], II.2, proposition 6). From this, it follows that the homotopy results of 4], section 6, suitably modified, hold for $R \mathcal{C D G} \mathcal{A}^{\circ}$ and $R \mathcal{C D} \mathcal{G} \mathcal{A}$ as well.

Definition 3.1. We shall say that an $R \mathrm{CDGA}$ (resp. $R \mathrm{CDGA}^{\circ}$ ) $A$ is formal if $A \approx H(A)$ in $H o(R \mathcal{C} \mathcal{D} \mathcal{G})$ (resp. $H o\left(R \mathcal{C D} \mathcal{G} \mathcal{A}^{\circ}\right)$ ).

If a functor $j: \mathcal{C}_{1} \rightarrow \mathcal{C}_{2}$ between two closed model categories preserves weak equivalences, then $X \approx Y$ in $H_{o} \mathcal{C}_{1}$ implies $j(X) \approx j(Y)$ in $H_{o} \mathcal{C}_{2}$. Thus if an algebra $A$ is formal as an $R \mathrm{CDGA}$, then it is formal as an $R \mathrm{CDGA}^{\circ}$, and as a $k$ CDGA, etc.

Suppose a smooth manifold $M$ has a smooth action of a compact Lie group $G$. The equivariant cohomology of $M$ may be computed by means of the Cartan complex of equivariant differential forms: $A_{G}^{\bullet}(M)=\left(\left(S \mathfrak{g}^{*} \otimes A^{\bullet}(M)\right)^{G}, d_{G}\right)$ where the differential, $d_{G}$, is zero on $S \mathfrak{g}^{*}$, and for $\alpha \in A^{\bullet}(M), d_{G} \alpha=d \alpha-\Sigma u_{i} \iota_{X_{i}} \alpha$, where 
the $\left\{X_{i}\right\}$ are fundamental vector fields of the action corresponding to a basis of $\mathfrak{g}$, and the $\left\{u_{i}\right\}$ are the corresponding algebra generators of $S \mathfrak{g}^{*}$, which are given degree two. If $M$ is just a topological space, we may compute the equivariant cohomology of $M$ by means of the $\mathbb{Q}$ CDGA $A_{G}^{\bullet}(M)$ of [2], when $G=S^{1}$. Alternatively, we could use the de Rham algebra of the Borel construction, $A^{\bullet}\left(E G \times{ }_{G} M\right)$ when $M$ is a manifold, or the Sullivan-de Rham algebra of the Borel construction when $M$ is not a manifold. We shall let $A_{G}^{\bullet}(M)$ possibly denote any of the above $k$ CDGA's, leaving it to the reader to interpret which model one prefers to use, as well as which ground field $k$. For a comprehensive treatment of equivariant de Rham theory, see [13.

Using either model, it is obvious how to obtain an $R$-algebra structure on $A_{G}^{\bullet}(M)$. It is induced by $R \stackrel{i}{\hookrightarrow} A_{G}^{\bullet}(p t$. $) \rightarrow A_{G}^{\bullet}(M)$, where the first map is a choice of minimal model for $A_{G}^{\bullet}(p t$.) in $k \mathcal{C D} \mathcal{G} \mathcal{A}$, and the second map is induced from the map $M \rightarrow\{p t$. $\}$. If we use the Cartan models, then the algebras $A_{G}^{\bullet}(M)$ are manifestly augmented over $R$ when the group action has a fixed point. This is because in the Cartan model, $A_{G}^{\bullet}(p t)=$.$R , and the inclusion of a fixed point gives$ a map $A_{G}^{\bullet}(M) \rightarrow A_{G}^{\bullet}(p t)=$.$R . However, if we use the Sullivan-de Rham complex$ of the Borel construction, then $A_{G}^{\bullet}(p t)=.A^{\bullet}(B G) \neq R$. Thus we must use a quasiisomorphic complex that is smaller and augmented over $R$. In [1], Allday shows that the complex $\eta^{-1}(R)$ is quasi-isomorphic to $A_{G}^{\bullet}(M)$, where $\eta: A_{G}^{\bullet}(M) \rightarrow A_{G}^{\bullet}(p t$.) is induced by the inclusion of a fixed point into $M$, and $R$ is embedded in $A_{G}^{\bullet}(p t$.) via $i$ as above. Clearly, $\eta^{-1}(R)$ is augmented over $R$, and is functorial for equivariant maps of $G$-spaces. We shall abuse notation and continue to write $A_{G}^{\bullet}(M)$, even when we may really mean $\eta^{-1}(R)$.

Let $\mathcal{G} \mathcal{T O P}$ denote the category of path-connected topological $G$-spaces with morphisms the equivariant maps. Then the under category $\{p t.\} \backslash \mathcal{G T O P}$ consists of "based $G$-spaces", which is the same thing as $G$-spaces with non-empty fixedpoint set and a choice of base point in the fixed-point set. Then $A_{G}^{\bullet}(-)$ gives a functor from $\mathcal{G} \mathcal{T O P}$ to $R \mathcal{C D G} \mathcal{A}^{o}$ and from $\{p t.\} \backslash \mathcal{G} \mathcal{T} \mathcal{O P}$ to $R \mathcal{C D G} \mathcal{A}$.

Definition 3.2. We shall say that a $G$-space $M$ is $G$-formal if $A_{G}^{\bullet}(M)$ is formal as an $R \mathrm{CDGA}^{\circ}$. A $G$-space $M$ with equivariant base point $p$ (i.e., a choice of fixed point $\left.p \in M^{G}\right)$ is $G$-formal at $p$ if $A_{G}^{\bullet}(M)$ is formal as an RCDGA, where $A_{G}^{\bullet}(M)$ is augmented via the inclusion of $p$ into $M$.

If we continue to define a minimal model of an $R$ CDGA as a connected cofibrant model $\mathcal{M}$ for which the induced differential on $\operatorname{ker} \varepsilon /(\operatorname{ker} \varepsilon)^{2}$ is zero, where $\varepsilon$ is an augmentation over $R$, then there may not be a minimal model for every algebra in $R \mathcal{C D G} \mathcal{A}$. An example is $S^{1}$ acting by rotations of $S^{2}$ about an axis. It is easy to see that there can be no minimal model for $A_{S^{1}}^{\bullet}\left(S^{2}\right)$ in $R C \mathcal{D G} \mathcal{A}$. However, there is a fairly canonical choice of cofibrant model for an $R$ CDGA. Let $R \rightarrow A$ be an $R \mathrm{CDGA}^{\circ}$. Then the map $R \rightarrow A$, viewed in $k \mathcal{C D G} \mathcal{A}$, may be factored as $R \rightarrow R \otimes_{k} \mathcal{M} \rightarrow A$ with the first map the inclusion, the latter map a quasiisomorphism, and $\mathcal{M}$ a minimal KS-complex, 14. Note that the differential on $R \otimes \mathcal{M}$ is not the tensor product differential; see the definition of a KS-complex (Definition 2.1). The map $R \rightarrow R \otimes \mathcal{M}$ is a minimal KS-extension, in particular a cofibration in $k \mathcal{C D G} \mathcal{A}$, and hence we see that $R \otimes \mathcal{M}$ is a cofibrant model for $A$ in $R \mathcal{C D G} \mathcal{A}^{\circ}$. Suppose $A$ is, moreover, an algebra in $R \mathcal{C D G} \mathcal{A}$, and let $\varepsilon: A \rightarrow R$ be its augmentation. Then composing $R \otimes \mathcal{M} \rightarrow A \stackrel{\varepsilon}{\rightarrow} R$ gives an $R$-augmentation for 
$R \otimes \mathcal{M}$. Thus, $R \otimes \mathcal{M}$ becomes a cofibrant model for $A$ in the category $R \mathcal{C D} \mathcal{G} \mathcal{A}$. As defined, it is unique up to isomorphism.

For those algebras of the form $A_{G}^{\bullet}(M)$ arising from a group action on the space $M$, this cofibrant model is more explicitly given by the Grivel-Halperin-Thomas theorem, which states that there is a commutative diagram

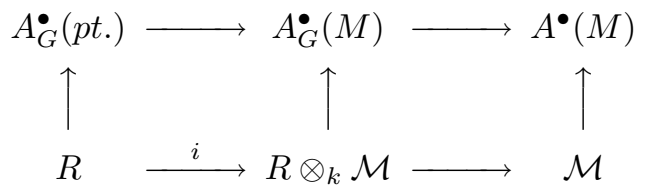

associated to the fibration $M \rightarrow E G \times{ }_{G} M \rightarrow B G$, where $\mathcal{M}$ is a minimal model for $M$, and the bottom row is a $\Lambda$-minimal $\Lambda$-extension, see [12], 14].

Definition 3.3. We shall refer to $R \otimes \mathcal{M}$ as the $G$-model of $A$, or just simply as the $G$-model of $M$, when $A=A_{G}^{\bullet}(M)$.

Sometimes we may choose to denote it by $\mathcal{M}_{G} \stackrel{\text { def }}{=} R \otimes \mathcal{M}$. Note that $R \otimes \mathcal{M}$ may fail to be minimal as a $k$ CDGA.

Following [1], 3], given a path-connected $G$-space $M$ with equivariant base point (i.e., a fixed point) $p$, the equivariant (pseudo-dual) $k$-homotopy groups are defined to be

$$
\Pi_{G, p}^{n}(M) \stackrel{\text { def }}{=} \pi^{n}(R \otimes \mathcal{M})=H^{n}\left(\operatorname{ker} \varepsilon /(\operatorname{ker} \varepsilon)^{2}\right),
$$

where $\varepsilon: R \otimes \mathcal{M} \rightarrow R$ is the $R$-algebra augmentation induced by the inclusion of $p$ into $M$, as above. The assignment $(M, p) \mapsto(R \otimes \mathcal{M}, \varepsilon)$ gives a functor from $\{p t.\} \backslash \mathcal{G} \mathcal{T} \mathcal{O P}$ to $H o(R \mathcal{C D G} \mathcal{A})$, and the equivariant pseudo-dual $k$-homotopy groups are functorial as well. Note that if $M$ is $G$-formal, then the equivariant pseudo-dual $k$-homotopy groups are determined by the equivariant cohomology ring of $M$.

The following lemma is useful for comparing the equivariant pseudo-dual $k$ homotopy groups to the ordinary pseudo-dual $k$-homotopy groups of the Borel construction.

Lemma 3.4. Let $A$ be an $R C D G A$ and let $R \otimes \mathcal{M}$ be the $G$-model for $A$. Then $R \otimes \mathcal{M}$ is minimal in $k \mathcal{C D} \mathcal{G} \mathcal{A}$.

Proof. We have the augmentation $\varepsilon: R \otimes \mathcal{M} \rightarrow R$, which is a map of $R \mathrm{CDGA}^{o}$ 's. The differential, $D$, on $R \otimes \mathcal{M}$ satisfies $D(r \otimes 1)=0$, for $r \in R$, and generally has the form $D(1 \otimes \alpha)=r \otimes 1+\sum r_{i} \otimes \alpha_{i}+1 \otimes d \alpha$, where $\alpha, \alpha_{i} \in \mathcal{M}, r, r_{i} \in R$ with $\operatorname{deg}(\alpha), \operatorname{deg}\left(\alpha_{i}\right), \operatorname{deg}(r), \operatorname{deg}\left(r_{i}\right)>0$, and where $d$ is the differential in $\mathcal{M}$. Now, $0=\varepsilon D(1 \otimes \alpha)=r+\sum r_{i} \varepsilon\left(\alpha_{i}\right)+\varepsilon(d \alpha)$. Since $d \alpha \in\left(\mathcal{M}^{+}\right)^{2}$, and $\varepsilon$ is an algebra map, it follows that $\sum r_{i} \varepsilon\left(\alpha_{i}\right)+\varepsilon(d \alpha) \in\left(R^{+}\right)^{2}$. Hence, we must have that $r=0$, and it follows that $R \otimes \mathcal{M}$ is minimal.

As an example, the pseudo-dual $k$-homotopy groups of the Borel construction of $S^{1}$ acting on $S^{2}$ do not distinguish the trivial action from a standard non-trivial one, whereas the equivariant pseudo-dual $k$-homotopy groups do.

\section{Generalities COnCERning $G$-Formality}

In this section we give some basic results about $G$-formality, including reduction to subgroups and the $G$-formality of products and wedges. 
We begin by noting that formality in the category $R \mathcal{C D G} \mathcal{A}^{o}$ is equivalent to formality in $k$ CDGA. In general, for two $R$-algebras $A$ and $B, A \approx B$ in $H o(k C \mathcal{C G A})$ does not imply that $A \approx B$ in $H o\left(R C D \mathcal{G} \mathcal{A}^{\circ}\right)$. Nevertheless, we have the following.

Lemma 4.1. Assume that $R \stackrel{j}{\rightarrow} A$ is an $R C D G A^{\circ}$ and that we give $H(A)$ the

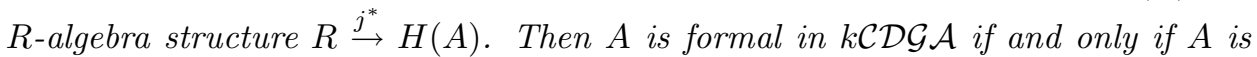
formal in $R \mathcal{C D G} \mathcal{A}^{o}$.

Proof. If $A$ is formal in $R \mathcal{C D} \mathcal{G} \mathcal{A}^{o}$, then it will be so in $k \mathcal{C D G} \mathcal{A}$, as we have noted above. Let us now assume that $A$ is formal in $k \mathcal{C D G} \mathcal{A}$. Let $\mathcal{N}$ be a minimal model for $A$ and let $R \otimes \mathcal{M}$ be the $G$-model for $A$. Then we have a commutative diagram of $k$ CDGA's

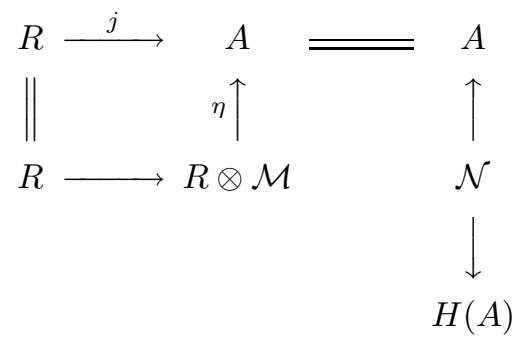

Since $R \otimes \mathcal{M}$ is cofibrant in $k \mathcal{C D} \mathcal{G} \mathcal{A}$, there exists a map, which is necessarily a quasiisomorphism, $R \otimes \mathcal{M} \rightarrow \mathcal{N}$ making the upper right square homotopy commute. This gives us a quasi-isomorphism $\varphi: R \otimes \mathcal{M} \rightarrow \mathcal{N} \rightarrow H(A)$. Then the map

$$
R \otimes \mathcal{M} \stackrel{\varphi}{\rightarrow} H(A) \stackrel{\left(\varphi^{*}\right)^{-1}}{\rightarrow} H(R \otimes \mathcal{M}) \stackrel{\eta^{*}}{\rightarrow} H(A)
$$

is a quasi-isomorphism and a map of $R$-algebras.

Remark 4.2. We note that this is not true for maps, however. That is, if $f: A \rightarrow B$ is a map of $R \mathrm{CDGA}^{o}$ 's, and $f$ is formal as a map of $k \mathrm{CDGA}^{\circ}$, then $f$ need not be a formal map of $R \mathrm{CDGA}^{o}$ 's.

In the category $R \mathcal{C D G} \mathcal{A}$, formality is a concept distinct from formality in the category $k \mathcal{C D G} \mathcal{A}$. In fact, it is easy to see that $M$ is $G$-formal at $p$ if and only if the map $i: B G \rightarrow E G \times_{G} M$ is a formal map, where $i$ is the map induced by the inclusion of $p$ into $M$.

Definition 4.3. Suppose that $G$ acts on a space $M$. Then the Serre spectral sequence associated with the fibration $M \rightarrow E G \times_{G} M \rightarrow B G$ is the same as the spectral sequence (from $E_{2}$ onwards) obtained from the $G$-model $R \otimes \mathcal{M}$ via the filtration $\mathcal{F}^{p}=R^{\geq p} \otimes \mathcal{M}$. If this spectral sequence degenerates at the $E_{2}$ term, then 11] refers to $M$ as being equivariantly formal. For obvious reasons, we wish to avoid this terminology; however, to conform as well to current trends, we shall say that $M$ is $e f$ when this spectral sequence degenerates at the $E_{2}$ term.

Proposition 4.4. Let $G$ act on a space $M$. Suppose that $K \subset G$ is a closed, connected subgroup. If $M$ is $G$-formal at $p$ (or $G$-formal) and ef, then $M$ is $K$ formal at $p$ (resp. $K$-formal). 
Proof. We first consider the case where $M$ is $G$-formal at $p$. The inclusion $K \subset G$ induces a pull-back diagram

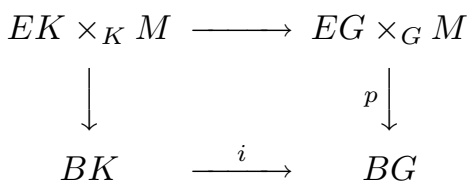

We shall denote $H^{\bullet}(B G)$ by $R_{G}$, and similarly for $R_{K}$. If we are using the Cartan complex of equivariant differential forms, then there is no problem with the proof. If we are using Allday's construction, $\eta^{-1}(R)$, as notated above, then we face the possibility that this construction may not be functorial with respect to changing the group. This is because there may not exist choices of minimal models so that $R_{G} \rightarrow R_{K} \rightarrow A^{\bullet}(B K)$ commutes with $R_{G} \rightarrow A^{\bullet}(B G) \stackrel{i}{\rightarrow} A^{\bullet}(B K)$. Then there would not exist an induced map $\eta^{-1}\left(R_{G}\right) \rightarrow \eta^{-1}\left(R_{K}\right)$.

This problem may be circumvented by the following procedure, as pointed out to us by C. Allday. Consider the diagram (6). Let $\tilde{Y}$ denote the mapping cylinder of the top row, and $Y$ the mapping cylinder of the bottom row. Then we have a commutative diagram

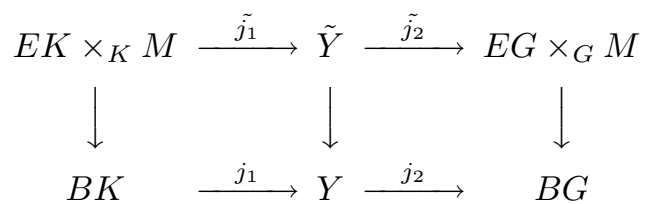

in which the maps $j_{1}, \tilde{j_{1}}$ induce surjections on differential forms and the maps $j_{2}, \tilde{j_{2}}$ induce quasi-isomorphisms on differential forms. It is easy to show that we may use $\tilde{Y}$ to form the complex $\eta^{-1}\left(R_{G}\right)$, as discussed in section 3, and that this complex will be quasi-isomorphic to $A_{G}^{\bullet}(M)$, and $G$-formal at $p$ if $M$ is $G$-formal at $p$. Moreover, we now may obtain a commutative diagram

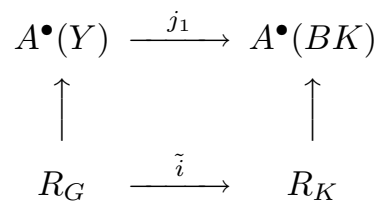

in which the vertical arrows are quasi-isomorphisms, since the map $A^{\bullet}(Y) \rightarrow$ $A^{\bullet}(B K)$ is onto. This follows by the result for $R \mathcal{C D G} \mathcal{A}$, which is the analog of 6.9 in 4 .

By Lemma A.1 of the appendix, there is a quasi-isomorphism of $k$ CDGA's

$$
\bar{B}\left(A^{\bullet}(B K), A^{\bullet}(B G), A_{G}^{\bullet}(M)\right) \rightarrow A_{K}^{\bullet}(M),
$$

where we are abusing notation in the event that we are using Allday's construction. Then, in either case, we obtain a quasi-isomorphism of $R_{K}$ CDGA's

$$
\bar{B}\left(R_{K}, R_{G}, A_{G}^{\bullet}(M)\right) \rightarrow A_{K}^{\bullet}(M) .
$$

The bar complex (10) is an $R_{K}$-algebra via the $R_{K}$ factor, and has an $R_{K}$-augmentation given by $\varepsilon\left(r_{K}, \alpha\right)=r_{K} \tilde{i}\left(\varepsilon_{G}(\alpha)\right)$, where $r_{K} \in R_{K}, \alpha \in A_{G}^{\bullet}(M)$, and $\varepsilon_{G}$ : 
$A_{G}^{\bullet}(M) \rightarrow R_{G}$ is the augmentation of $M$ for the action of $G$. By the assumption of $G$-formality, we get a commuting diagram whose vertical arrows are quasiisomorphisms:

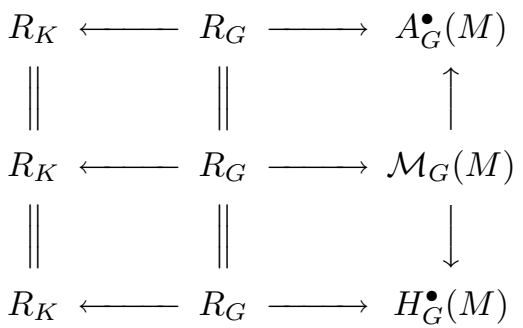

Then we obtain the following sequence of maps, which are seen to be $R_{K}$ CDGA quasi-isomorphisms by standard comparison theorems for their associated Eilenberg-Moore spectral sequences:

$$
\bar{B}\left(R_{K}, R_{G}, A_{G}^{\bullet}(M)\right) \leftarrow \bar{B}\left(R_{K}, R_{G}, \mathcal{M}_{G}(M)\right) \rightarrow \bar{B}\left(R_{K}, R_{G}, H_{G}^{\bullet}(M)\right) .
$$

Now the bar complex $\bar{B}\left(R_{K}, R_{G}, H_{G}^{\bullet}(M)\right)$ has only the single differential $\delta$, and computes $\operatorname{Tor}_{R_{G}}\left(R_{K}, H_{G}^{\bullet}(M)\right)$. Since $M$ is ef, $H_{G}^{\bullet}(M)$ is a free $R_{G}$-module. Hence we have that $\bar{B}\left(R_{K}, R_{G}, H_{G}^{\bullet}(M)\right)$ • is acyclic in bar degrees greater than zero, and the projection to cohomology

$$
\begin{aligned}
\bar{B}\left(R_{K}, R_{G}, H_{G}^{\bullet}(M)\right) \bullet & \rightarrow \bar{B}\left(R_{K}, R_{G}, H_{G}^{\bullet}(M)\right)_{0} \\
& \rightarrow H_{\bullet}\left(\bar{B}\left(R_{K}, R_{G}, H_{G}^{\bullet}(M)\right) \bullet\right. \\
& \approx H_{K}^{\bullet}(M)
\end{aligned}
$$

is an $R_{K}$ CDGA quasi-isomorphism.

The case where we consider $M$ to be $G$-formal in the category $R_{G} \mathcal{C} \mathcal{G} \mathcal{A}^{o}$ is similar.

Corollary 4.5. Let $G$ act on a space $M$. Suppose that $M$ is $G$-formal at $p$ (or $G$-formal) and ef. Then $M$ is formal in $k \mathcal{C D G} \mathcal{A}$.

Proof. Just take $K$ to be the identity subgroup in Proposition 4.4

Remark 4.6. If we use Remark A.5 of the appendix, then we can see that a $K$-model for $M$ is given by $\bar{B}_{R_{G}}\left(R_{K}, R_{G}, \mathcal{M}_{G}(M)\right)=R_{K} \otimes_{R_{G}} \mathcal{M}_{G}(M)$.

In line with the general theme of considering maximal tori in compact, connected Lie groups, we have the following fact, which is due to C. Allday. We say that a space $M$ is of finite type if $H^{i}(M)$ is a finite-dimensional $k$-vector space for all $i$.

Proposition 4.7. Let $G$ act on $M$, and let $T \subset G$ be a maximal torus. If $M$ is $G$-formal ( $G$-formal at $p$ ), then $M$ is $T$-formal (resp. T-formal at $p$ ). Moreover, if $M$ is a space of finite type, $p \in M^{G}$, and $M$ is $T$-formal at $p$, then $M$ is $G$-formal at $p$.

Proof. We can already see that $G$-formal implies $T$-formal by the proof of Proposition 4.4 We only need the fact that now $R_{T}$ is a free $R_{G}$-module, which follows from the well-known fact that as $R_{G}$-modules, $R_{T} \approx R_{G} \otimes H^{\bullet}(G / T)$.

Showing that $T$-formal at $p$ implies $G$-formal at $p$ may be achieved by imitating the proof that $A \otimes K$ being formal implies $A$ is formal, for $K$ an extension field of $k$, which is corollary 6.9 of [15]. We omit the details, but mention the setup. First, 
we see by Remark 4.6 that a $T$-model for $A_{T}^{\bullet}(M)$ is given by $R_{T} \otimes_{R_{G}} \mathcal{M}_{G}(M)$ with differential $1 \otimes D_{G}$, where $D_{G}$ is the differential for the $G$-model $\mathcal{M}_{G}(M)$. Thus it suffices to show that if $R_{T} \otimes_{R_{G}} \mathcal{M}_{G}(M)$ is formal as an $R_{T}$ CDGA, then $\mathcal{M}_{G}(M)$ is formal as an $R_{G}$ CDGA.

It turns out that the constructions of bigraded and filtered models of the relevant algebras, as in [15, give models in the category $R \mathcal{C D} \mathcal{G} \mathcal{A}$. The proof of corollary 6.9 may be imitated without too much difficulty.

Proposition 4.8. Suppose that $X$ and $Y$ are $G$-spaces, both of which are $G$-formal (or assume $X$ is $G$-formal at $p$ and $Y$ is $G$-formal at $q$ ), and suppose that one or both of them is ef. Then $X \times Y$ is $G$-formal (resp. $G$-formal at $(p, q))$ for the diagonal action of $G$.

Proof. The pull-back diagram

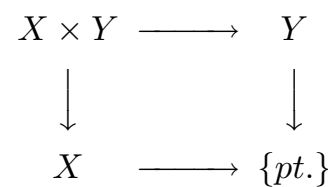

gives rise to a pull-back diagram

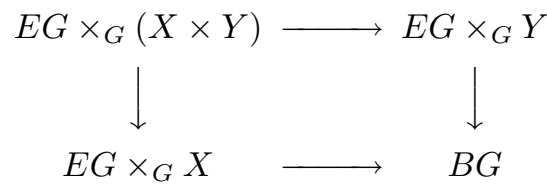

Then we obtain an $R \mathrm{CDGA}^{\circ}$ quasi-isomorphism

$$
\bar{B}\left(A_{G}^{\bullet}(X), A_{G}^{\bullet}(\{p t .\}), A_{G}^{\bullet}(Y)\right) \stackrel{\theta}{\rightarrow} A_{G}^{\bullet}(X \times Y)
$$

by Lemma A.3 of the appendix. If $X$ and $Y$ both have fixed points, then so will their product $X \times Y$. In that case, $\theta$ is a quasi-isomorphism of $R$ CDGA's by Lemma A.3 of the appendix. Furthermore,

$$
\bar{B}\left(A_{G}^{\bullet}(X), R, A_{G}^{\bullet}(Y)\right) \rightarrow \bar{B}\left(A_{G}^{\bullet}(X), A_{G}^{\bullet}(\{p t .\}), A_{G}^{\bullet}(Y)\right)
$$

is an $R \mathrm{CDGA}^{\circ}$ (RCDGA) quasi-isomorphism. Since $X$ and $Y$ are $G$-formal, we get $R \mathrm{CDGA}^{\circ}(R \mathrm{CDGA})$ quasi-isomorphisms of bar complexes

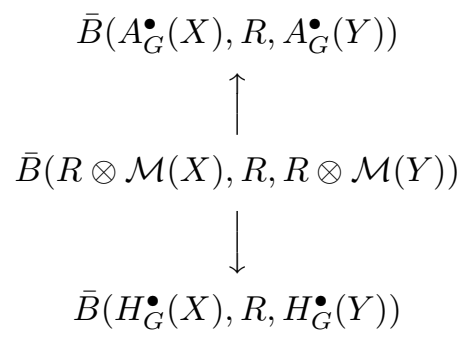

by standard arguments comparing the associated Eilenberg-Moore spectral sequences.

Since one or both of $X, Y$ is ef, just as in the proof of Proposition 4.4, the bar complex $\left(\bar{B}\left(H_{G}^{\bullet}(X), R, H_{G}^{\bullet}(Y)\right) \bullet ; \delta\right)$ is acyclic in degrees greater than zero with respect to the bar grading, and the projection to its cohomology is an $R \mathrm{CDGA}^{o}$ ( $R$ CDGA) quasi-isomorphism. 
Proposition 4.9. Let $X$ and $Y$ be $G$-spaces whose fixed-point sets are non-empty. Picking base points $p \in X^{G}$ and $q \in Y^{G}$, we may form the wedge $X \vee Y$ along these base points. Then $G$ acts on $X \vee Y$. If $X$ is $G$-formal at $p$ and $Y$ is $G$-formal at $q$, then $X \vee Y$ is $G$-formal at the join of $p$ and $q$.

Proof. Let $\varepsilon_{X}, \varepsilon_{Y}$ denote the augmentations of equivariant differential forms, and let $i_{X}, i_{Y}$ denote the inclusions of $X, Y$ into $X \vee Y$. Then Mayer-Vietoris gives a short exact sequence

$$
0 \rightarrow A_{G}^{\bullet}(X \vee Y) \stackrel{i_{X}+i_{Y}}{\rightarrow} A_{G}^{\bullet}(X) \oplus A_{G}^{\bullet}(Y) \stackrel{\varepsilon_{X}-\varepsilon_{Y}}{\rightarrow} R \rightarrow 0 .
$$

Thus $i_{X}+i_{Y}$ induces an isomorphism

$$
A_{G}^{\bullet}(X \vee Y) \approx A_{G}^{\bullet}(X) \bigoplus_{R} A_{G}^{\bullet}(Y)=\operatorname{ker}\left\{\varepsilon_{X}-\varepsilon_{Y}\right\}
$$

Moreover, since $\varepsilon_{X}$, say, induces a surjection in cohomology, the associated long exact sequence splits into short exact sequences, and thus

$$
H_{G}^{\bullet}(X \vee Y) \approx H_{G}^{\bullet}(X) \oplus_{R} H_{G}^{\bullet}(Y) .
$$

Since $X$ and $Y$ are $G$-formal, we have maps $A_{G}^{\bullet}(X) \leftarrow \mathcal{M}_{G}(X) \rightarrow H_{G}^{\bullet}(X)$ which are quasi-isomorphisms of $R$ CDGA's, and similarly for $Y$. So we have a commutative diagram whose rows are short exact sequences:

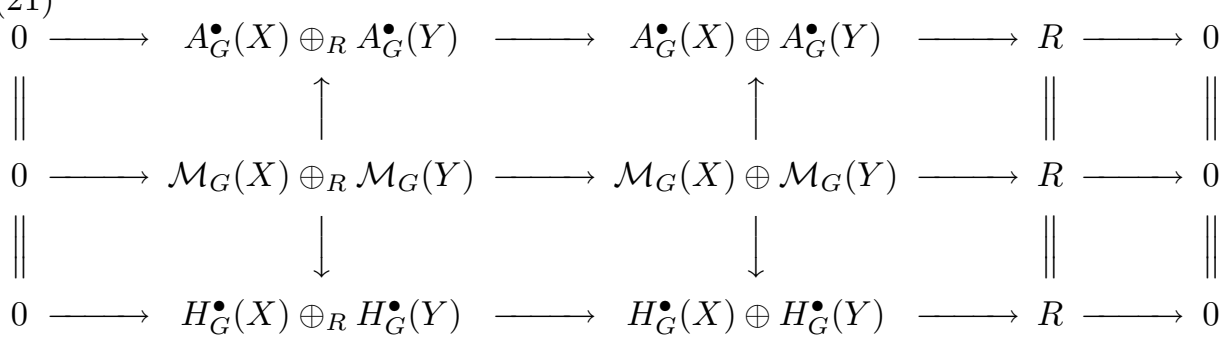

Then we obtain maps between the associated long exact sequences in cohomology. By the 5-lemma, it follows that the maps

$$
A_{G}^{\bullet}(X) \oplus_{R} A_{G}^{\bullet}(Y) \leftarrow \mathcal{M}_{G}(X) \oplus_{R} \mathcal{M}_{G}(Y) \rightarrow H_{G}^{\bullet}(X) \oplus_{R} H_{G}^{\bullet}(Y)
$$

are quasi-isomorphisms. It is easy to check that these maps are compatible with augmentations and the $R$-algebra structure, so are $R$ CDGA quasi-isomorphisms.

\section{EXAmples of $G$-Formal SPACES}

In this section we give some examples of $G$-formal spaces.

5.1. Compact Kähler manifolds. Let $M$ be a compact Kähler manifold, and $G$ a compact, connected Lie group acting on $M$ by holomorphic transformations. We introduce equivariant holomorphic cohomology groups. Since $M$ is a complex manifold, the complex-valued differential forms on $M$ are bigraded in the usual way. We shall denote $S \mathfrak{g}^{*} \otimes_{\mathbb{R}} \mathbb{C}$ by simply $S \mathfrak{g}^{*}$. Then we define the equivariant Dolbeault cohomology to be the cohomology of the complex

$$
\left(\left[S \mathfrak{g}^{*} \otimes_{\mathbb{C}} A^{p, \bullet}(M)\right]^{G} ; \bar{\partial}+\sum u_{i} \iota_{i}\right) .
$$

Here $Z_{i}$ is the holomorphic vector field on $M$ which comes about by splitting the fundamental vector field $X_{i}=Z_{i}+\bar{Z}_{i}$ into its holomorphic and anti-holomorphic 
components. The generators $u_{i} \in S \mathfrak{g}^{*}$ are given bidegree $(1,1)$. The operators act in a similar way as for the ordinary equivariant cohomology. We shall denote the $q$ th cohomology of this complex by $H_{G}^{p, q}(M)$.

The following theorem was proved in [17] and independently established in [25].

Theorem 5.1. Suppose that $M$ is a compact Kähler manifold endowed with a holomorphic action of a compact, connected Lie group $G$, and suppose that $M$ is ef for the action of $G$. Then $M$ is $G$-formal. If $M^{G} \neq \varnothing$, then $M$ is $G$-formal at any fixed point.

Proof. The Cartan complex is $\left(A_{G}^{\bullet}(M), d_{G}\right)=\left(\left[S \mathfrak{g}^{*} \otimes A^{\bullet}(M ; \mathbb{C})\right]^{G} ; d+\sum u_{i} \iota_{X_{i}}\right)$. Let $X_{i}=Z_{i}+\bar{Z}_{i}$ be the splitting of the fundamental vector field $X_{i}$ into its holomorphic and anti-holomorphic parts. The differential $d=\partial+\bar{\partial}$ also splits. Hence we may split the equivariant differential as $d+\sum u_{i} \iota X_{i}=\left(\bar{\partial}+\sum u_{i} \iota_{Z_{i}}\right)+$ $\left(\partial+\sum u_{i} \iota \bar{Z}_{i}\right)$. The complex $\left[S \mathfrak{g}^{*} \otimes A_{G}^{\bullet}(M ; \mathbb{C})\right]^{G}$ is bigraded by giving $u_{i} \in S \mathfrak{g}^{*}$ bidegree $(1,1)$, and taking the usual bigrading on $A^{\bullet}(M ; \mathbb{C})$. It is easy to show that

$$
\left(\left[S \mathfrak{g}^{*} \otimes A^{\bullet \bullet \bullet}(M ; \mathbb{C})\right]^{G} ;\left(\bar{\partial}+\sum u_{i} \iota Z_{i}\right),\left(\partial+\sum u_{i} \iota \bar{Z}_{i}\right)\right)
$$

is a first quadrant double complex. Accordingly we have two canonical filtrations of this complex. We claim that the spectral sequences corresponding to both of them degenerate at the $E_{1}$ term, and moreover are $n$-opposite, meaning that ${ }^{\prime} F^{p} \oplus^{\prime \prime} F^{q} \approx$ $H^{n}$ for $p+q-1=n$. Formality for $A_{G}^{\bullet}(M)$ then follows owing to the results in [6], sections 5 and 6 .

Let us consider the filtration in which we take $\bar{\partial}+\sum u_{i} \iota_{Z_{i}}$ cohomology first. This is the Dolbeault equivariant cohomology defined above. It itself forms a first quadrant double complex with the two differentials $\bar{\partial}$ and $\sum u_{i} \iota_{Z_{i}}$. Let us filter so that we take the $\bar{\partial}$ cohomology first. Then the $E_{1}$ term for the equivariant Dolbeault complex is (additively)

$$
\begin{aligned}
H\left(\left[S \mathfrak{g}^{*} \otimes A^{\bullet}(M)\right]^{G} ; \bar{\partial}\right) & \approx\left[H\left(S \mathfrak{g}^{*} \otimes A^{\bullet}(M) ; \bar{\partial}\right)\right]^{G} \approx\left(S \mathfrak{g}^{*} \otimes H \bullet \bar{\partial}(M)\right)^{G} \\
& \approx\left(S \mathfrak{g}^{*}\right)^{G} \otimes H \cdot \overline{\bar{\partial}}(M) \approx H^{\bullet}(B G) \otimes H \cdot \bar{\partial}(M) .
\end{aligned}
$$

Now by ordinary Hodge theory for compact Kähler manifolds, this last is isomorphic to $H^{\bullet}(B G) \otimes H^{\bullet}(M)$. But now there can be no further non-trivial differentials in the spectral sequence, by the assumption that $M$ is ef. This result follows analogously for the other filtration, which is just the complex conjugate of this one. Furthermore, it is easy to see that the two filtrations are $n$-opposite.

Hence we have a " $\partial_{G} \bar{\partial}_{G}$-lemma" for the equivariant differential forms, where we mean by $\bar{\partial}_{G}$ the equivariant Dolbeault operator as defined above. Formality follows via the sequence of $\mathbb{C} C D G A$ quasi-isomorphisms

$$
A_{G}^{\bullet}(M) \hookleftarrow \operatorname{ker}\left(\bar{\partial}_{G}\right) \rightarrow H_{G}^{\bullet}(M),
$$

which are the inclusion and projection, respectively. These maps are maps of $R$ algebras, and moreover, it follows that for equivariant holomorphic maps between $M$ and $N$, we get a commutative diagram linking the sequence (27) for $M$ to the analogous sequence for $N$. In particular, if the action of $G$ on $M$ has fixed points, then the inclusion of one (chosen as an equivariant base point) gives augmentations so that the sequence (27) commutes with augmentations. That is, $M$ is $G$-formal in $R \mathcal{C D G} \mathcal{A}$. 
Corollary 5.2. Suppose that $M$ is a compact Kähler manifold endowed with a holomorphic action of a compact, connected Lie group $G$. Assume that $M^{G} \neq \varnothing$. Then $M$ is $G$-formal at any fixed point.

Proof. Let $p \in M^{G}$. Let $T \subset G$ be a maximal torus. Then $M^{T} \neq \varnothing$, and a theorem of Blanchard says that $M$ is ef for the action of $T$; see [9], Chapter XII, theorem 6.2. By Theorem [5.1, $M$ is $T$-formal at $p$. By Proposition 4.7, $M$ is $G$-formal at $p$.

Remark 5.3. The proof of Theorem 5.1 implies an equivariant Hodge decomposition

$$
H_{G}^{n}(M) \approx \bigoplus_{p+q=n} H_{G}^{p, q}(M) .
$$

5.2. Elliptic spaces. We recall that an elliptic space $M$ is a space such that both $H^{\bullet}(M ; k)$ and $V$ are finite-dimensional $k$-vector spaces, where $\mathcal{M}(M)=\Lambda(V)$ is a minimal model for $M$. We shall use the following result of [19].

Proposition 5.4 (Lupton). Let $F \rightarrow E \rightarrow B$ be a fibration in which $F$ is formal and elliptic, and $B$ is formal and simply-connected. If the Serre spectral sequence of the fibration degenerates at the $E_{2}$ term, then $E$ is formal also.

Theorem 5.5. Let $M$ be an elliptic $G$-space. If $M$ is formal and ef, then $M$ is $G$-formal. If $M^{G} \neq \varnothing$, then $M$ is $G$-formal at any fixed point.

Proof. We have the fibration $M \rightarrow E G \times_{G} M \rightarrow B G$. Then Proposition 5.4 implies that $A_{G}^{\bullet}(M)$ is formal as a $k$ CDGA. The proof of Lupton's proposition works (adapting to our situation) by finding a model for $A_{G}^{\bullet}(M)$ of the form $R \otimes \mathcal{M}$ that is bigraded as a $k$ CDGA. Here, $\mathcal{M}$ is the bigraded (minimal) model of $M$. Elements of $R$ are in degree zero for the second grading, so that $(R \otimes \mathcal{M})_{0}=R \otimes(\mathcal{M})_{0}$. It is shown that with respect to the second grading we have $H_{+}(R \otimes \mathcal{M})=0$, and hence the projection to cohomology

$$
R \otimes \mathcal{M} \rightarrow(R \otimes \mathcal{M})_{0} \rightarrow H_{G}^{\bullet}(M)
$$

is a quasi-isomorphism. Clearly, this is a map of $R$-algebras. Moreover, if $M^{G} \neq \varnothing$, then this map commutes with the augmentations over $R$. This follows because first the map $R \otimes \mathcal{M} \rightarrow(R \otimes \mathcal{M})_{0}$ commutes with augmentations. Second, since the augmentation $\varepsilon: R \otimes \mathcal{M} \rightarrow R$ is a map of $R$ CDGA's, $\varepsilon(d \alpha)=0$ for all $\alpha$, so that the map $(R \otimes \mathcal{M})_{0} \rightarrow H_{G}^{\bullet}(M)$ commutes with augmentations.

Corollary 5.6. Let $M$ be an elliptic space. Suppose that a torus $T$ acts on $M$ with $M^{T} \neq \varnothing$. Suppose further that one of the components of the fixed-point set, say $M_{i}^{T}$, satisfies $H^{\text {odd }}\left(M_{i}^{T}\right)=0$. Then $M$ is $T$-formal at any fixed point.

Proof. Since $M$ is elliptic, it follows (via localization and localization for equivariant rational homotopy [3]) that each component of the fixed-point set is elliptic and $\chi_{\pi}(M)=\chi_{\pi}\left(M_{i}^{T}\right)$, where $\chi_{\pi}$ is the homotopy Euler characteristic. But Halperin has shown that for elliptic spaces the conditions $H^{\text {odd }}=0$ and $\chi_{\pi}=0$ are equivalent, and moreover such spaces are formal. Thus $0=\chi_{\pi}\left(M_{i}^{T}\right)=\chi_{\pi}(M)$. Hence $M$ is formal and $H^{\text {odd }}(M)=0$. But this latter condition implies that $M$ is ef. So we may apply Theorem 5.5

Remark 5.7. Suppose $G$ acts on a simply-connected space $M$ with non-empty fixedpoint set. Then by picking a base point in the fixed-point set, we obtain an action 
of $G$ on the space of based loops in $M$, denoted $\Omega M$. Since the cohomology of $\Omega M$ is free, we see that $\Omega M$ will be $G$-formal if $\Omega M$ is ef. (Lupton's proof could be extended to this case, as well.) If $G=T$ is a torus, and $M$ is elliptic, then the condition that $\Omega M$ is ef is equivalent to the $G$-model $R \otimes \mathcal{M}(M)$ being minimal in the category $R \mathcal{C D} \mathcal{G} \mathcal{A}$; see [3], 3.3.15.

5.3. Miscellanea. Next we shall give a few extra examples of $G$-formality.

Theorem 5.8. Let $M$ be a space with minimal model $\mathcal{M}=\bigwedge(V)$. Suppose that $d x=0$ for all $x \in V^{\text {even }}$ such that $\operatorname{deg}(x)<\operatorname{dim} M$. Suppose further that the circle $S^{1}=T$ acts on $M$, that $M$ is ef, and that each component of the fixed-point set is formal and satisfies $H^{\text {odd }}\left(M^{T}\right)=0$. Then $M$ is $T$-formal at any fixed point.

Proof. Since $M$ is ef, the Serre spectral sequence for the fibration $M \rightarrow E T \times_{T} M \rightarrow$ $B T$ degenerates at the $E_{2}$ term. (Note that by the localization theorem, this implies that $M^{T} \neq \varnothing$.) By a standard change of basis argument, we may assume that in the $T$-model $(R \otimes \mathcal{M}, D)$ we have $D x=0$, for $x \in V^{\text {even }}$ such that $\operatorname{deg}(x)<\operatorname{dim} M$. Let $i: M^{T} \hookrightarrow M$ denote the inclusion of the fixed-point set. Then we have maps of $R$ CDGA's (actually, the algebras on the right-hand side of the diagram do not satisy $H^{0}=k$, but this will not present any problems)

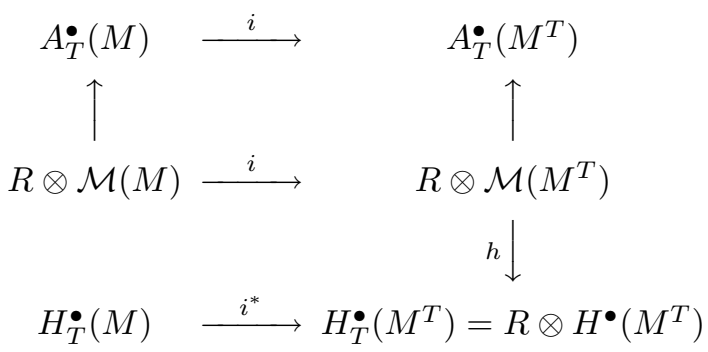

where $h$ is a quasi-isomorphism since $M^{T}$ is formal. Since $M$ is ef, the map $i^{*}$ is an injection. We claim that $h i(R \otimes \mathcal{M}(M)) \subseteq i^{*}\left(H_{T}^{\bullet}(M)\right)$. Since the maps are algebra maps, it suffices to check this on algebra generators. Since $M$ is ef, the localization theorem shows that $i^{*}$ is an isomorphism in degrees $\geq \operatorname{dim} M$. Also if $\alpha \in R \otimes \mathcal{M}(M)$ has odd degree, then $h i(\alpha)=0$, since $H_{T}^{\text {odd }}\left(M^{T}\right)=0$ by assumption. So it suffices to check the claim on algebra generators of $R \otimes \mathcal{M}(M)$ of even degree less than $\operatorname{dim} M$. Let $\alpha$ be such a generator. If $\alpha \in R$, then the claim is obviously true. If $\alpha \in \mathcal{M}(M)$, then by assumption $D \alpha=0$. Then $h i(\alpha)=[i(\alpha)]=j([\alpha])$. Thus we have a map

$$
R \otimes \mathcal{M}(M) \stackrel{h i}{\rightarrow} H_{T}^{\bullet}(M),
$$

which is a quasi-isomorphism of $R$ CDGA's.

Corollary 5.9. Let $M$ be a simply-connected space with minimal model $\bigwedge(V)$. Suppose that $d x=0$ for all $x \in V^{\text {even }}$ such that $\operatorname{deg}(x)<\operatorname{dim} M$. Suppose further that a torus $T$ acts on $M$, that $M$ is ef, and that each component of the fixed-point set is formal and satisfies $H^{\text {odd }}\left(M^{T}\right)=0$. Then $M$ is formal in $k \mathcal{C D G} \mathcal{A}$. 
Proof. First of all, there is a subcircle $S^{1} \subset T$ such that $M^{S^{1}}=M^{T}$. The inclusion of this circle $S^{1} \hookrightarrow T$ induces a pull-back diagram:

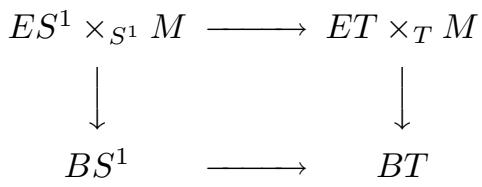

Since the action of $T$ is ef, the Serre spectral sequence for the fibration on the right degenerates at the $E_{2}$ term. But then the same is true for the pull-back fibration. Hence the $S^{1}$ action is ef as well. Now the result follows from Theorem 5.8 and Corollary 4.5

Corollary 5.10. Let $M^{4}$ be a space such that $H^{\text {odd }}(M)=0$ and $\operatorname{dim} M=4$. Suppose that a circle $S^{1}=T$ acts on $M$. Then $M$ is $T$-formal at any fixed point.

Proof. We have that $H^{o d d}(M)=0$, so that $M$ is ef. Then $M^{T} \neq \varnothing$. By localization, $H^{\text {odd }}\left(M^{T}\right)=0$. But path-connected spaces with $H^{1}=0$ of dimension less than or equal to 4 are formal; so each component of $M^{T}$ is formal. The result follows by Theorem 5.8.

Remark 5.11. A simple example of an $S^{1}$-space satisfying the conditions of Theorem 5.8, but which is not Kähler or elliptic, is the following. Let $S^{1}$ act on $S^{4}$ so that the fixed-point set consists of two isolated points. Extend this to a diagonal action of $S^{1}$ on $S^{4} \times S^{4}$. Then, removing a neighborhood of a fixed point, we may form the connected sum $S^{4} \times S^{4} \# S^{4} \times S^{4}$. This manifold then inherits an $S^{1}$ action with 6 isolated fixed points. It is not elliptic, and not even symplectic, since $H^{2}=0$. It is easy to check that it satisfies the conditions of Theorem [5.8, so is $S^{1}$-formal. (This can also be seen by proving that the connected sum (made in an equivariant setting) of $G$-formal spaces is again $G$-formal, which we have omitted.)

We conclude this section with two examples that do not involve the condition of $M$ being ef.

Lemma 5.12. Let $M$ be a simply-connected compact manifold. Suppose that $G$ acts freely on $M$ and $\operatorname{dim} G \geq \operatorname{dim} M-6$. Then $M$ is $G$-formal.

Proof. Since $G$ acts freely, $M / G$ is a simply-connected manifold of dimension 6 or less. Hence $M / G$ is formal [21]. So $E G \times_{G} M$ is formal.

Remark 5.13. Suppose, in the situation of Lemma [5.12, we have that $\operatorname{dim} M-6>$ $\operatorname{rank}(G)$. Let $T \subset G$ be a maximal torus. Then by Proposition 4.7, $M / T$ is a simply-connected manifold of dimension greater than 6 which is formal.

Lemma 5.14. Let $M$ be a simply-connected elliptic space. Suppose that $G$ acts almost freely on $M$ (meaning all isotropy groups are finite), and $\operatorname{rank}(G)=$ $-\chi_{\pi}(M)$. Then $M$ is $G$-formal.

Proof. Since $M$ and $B G$ have finite-dimensional pseudo-dual rational homotopy, so does $E G \times{ }_{G} M$, as may be seen by considering the fibration $M \rightarrow E G \times{ }_{G} M \rightarrow B G$. Since $G$ acts almost freely, $H^{\bullet}\left(E G \times{ }_{G} M\right)$ is finite-dimensional as well. Furthermore,

$$
\chi_{\pi}\left(E G \times_{G} M\right)=\chi_{\pi}(M)+\chi_{\pi}(B G)=-\operatorname{rank}(G)+\operatorname{rank}(G)=0 .
$$

Thus $E G \times_{G} M$ is elliptic with $\chi_{\pi}=0$, so is formal. 


\section{An Application}

In this section we give an application of $G$-formality. We will show that the computation of the equivariant cohomology of loop spaces simplifies considerably when the space is $G$-formal.

Let us consider a simply-connected space $M$. Suppose that $G$ acts on $M$ with non-empty fixed-point set. Let $p \in M^{G}$ be a choice of base point. Then we get an action of $G$ on the loops in $M$ based at $p, \Omega(M ; p)$, which we shall often abbreviate as $\Omega M$. Let $P(M ; p)$ be the space of paths in $M$, based at $p$. Then we have the fibration

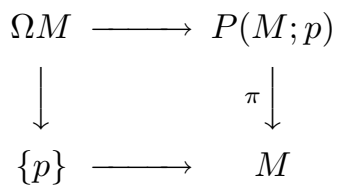

where $\pi$ is the map sending a path $\gamma(t)$ to its value at time $1, \gamma(1)$. Moreover, the $G$-action induces a pull-back diagram of fibrations

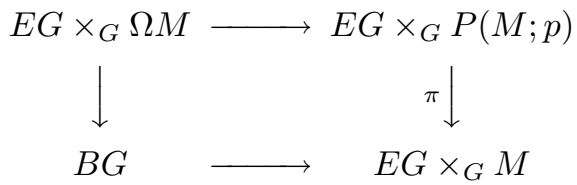

Hence there is a quasi-isomorphism of $R$ CDGA's

$$
\theta: \bar{B}\left(R, A_{G}^{\bullet}(M), A_{G}^{\bullet}(P(M ; p)) \rightarrow A_{G}^{\bullet}(\Omega(M))\right.
$$

by Lemma A.3 of the appendix. Now the inclusion of $\{p\}$ into $P(M ; p)$ followed by $\pi$ is the inclusion of $\{p\}$ into $M$. These maps are equivariant, so induce their analogs on the Borel constructions. Hence we get an RCDGA quasi-isomorphism

$$
\bar{B}\left(R, A_{G}^{\bullet}(M), R\right) \leftarrow \bar{B}\left(R, A_{G}^{\bullet}(M), A_{G}^{\bullet}(P(M ; p)) .\right.
$$

Proposition 6.1. Let $G$ act on a simply-connected space $M$ with non-empty fixedpoint set, so that $G$ acts on $\Omega M$. Suppose that $M$ is $G$-formal. Then there is an isomorphism of $R$-algebras

$$
H_{G}^{\bullet}(\Omega M) \approx \operatorname{Tor}_{H_{G}^{\bullet}(M)}(R, R) .
$$

Proof. We have that $A_{G}^{\bullet}(\Omega M)$ is quasi-isomorphic to $\bar{B}\left(R, A_{G}^{\bullet}(M), R\right)$ (via a sequence of $R C D G A$ quasi-isomorphisms). The assumption of $G$-formality means we have a commuting diagram of $R$-algebras

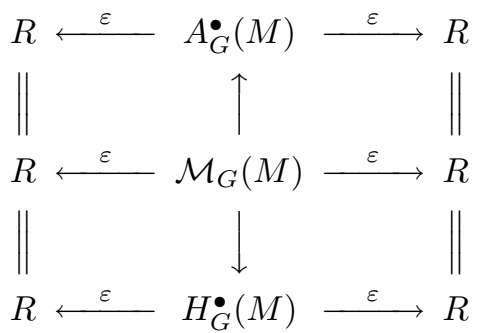

We obtain $R$ CDGA quasi-isomorphisms

$$
\bar{B}\left(R, A_{G}^{\bullet}(M), R\right) \leftarrow \bar{B}\left(R, \mathcal{M}_{G}(M), R\right) \rightarrow \bar{B}\left(R, H_{G}^{\bullet}(M), R\right) .
$$


This follows by standard comparison theorems for the Eilenberg-Moore spectral sequences associated to the bar complexes. Thus we have that $\bar{B}\left(R, H_{G}^{\bullet}(M), R\right)$ is quasi-isomorphic to $A_{G}^{\bullet}(\Omega M)$ (via a sequence of $R$ CDGA quasi-isomorphisms). But the cohomology of $\bar{B}\left(R, H_{G}^{\bullet}(M), R\right)$ is $\operatorname{Tor}_{H_{G}^{\bullet}(M)}(R, R)$.

Remark 6.2. We can always choose any resolution to compute Tor. But we note that we may always use the bar resolution, and using Lemma A.4 of the appendix, we see that when $M$ is $G$-formal, $H_{G}^{\bullet}(\Omega M)$ may be computed via the (single) complex

$$
\left(\bar{B}_{R}\left(R, H_{G}^{\bullet}(M), R\right) ; \delta\right) .
$$

Remark 6.3. We could also obtain analogous results for the equivariant cohomology of the free loop space $L M$.

\section{An Example}

In this section we compute an example of the equivariant cohomology of the based-loop space using the normalized bar complex over $R$ of Remark 6.2

7.1. Example: $S^{1}$ acting on $\Omega S^{2}$. The circle $S^{1}$ acts on the 2 -sphere $S^{2}$ by rotations about an axis, say the $z$-axis when $S^{2}$ is the unit sphere in $\mathbb{R}^{3}$. This action is holomorphic and Hamiltonian. Thus by Theorem 5.1, $S^{2}$ is $G$-formal $\left(G=S^{1}\right)$. It is easy to show that the equivariant cohomology ring is

$$
H_{S^{1}}^{\bullet}\left(S^{2} ; k\right) \approx k[x, u] /(x+u)(x-u),
$$

where the degree of $x$ and $u$ is two, and $R=k[u]$ acts as multiplication by $u$.

The fixed-point set, $F$, consists of the north and south poles. We shall write $F=\{N, S\}$. Let $\Omega S^{2}$ be loops based at the north pole. Then $S^{1}$ acts on $\Omega S^{2}$. Then the equivariant cohomology of the based loops, $H_{S^{1}}\left(\Omega S^{2}\right)$, may be computed as the cohomology of the bar complex

$$
\left(\bar{B}_{k[u]}\left(k[u], \frac{k[x, u]}{\left(x^{2}-u^{2}\right)}, k[u]\right) ; \delta\right) .
$$

Let $\omega$ be the symplectic form on $S^{2}$. Then $x$ is represented by the form $\omega-$ $u f \in A_{S^{1}}\left(S^{2}\right)$, and $u$ is represented by the form $u \in A_{S^{1}}\left(S^{2}\right)$, using the Cartan complex of equivariant differential forms. Here, $f$ is the moment map which sends a point on $S^{2} \subset \mathbb{R}^{3}$ to its $z$-component. Then the inclusion of the north pole $\{N\}$ into $S^{2}$ induces the augmentation $H_{S^{1}}^{\bullet}\left(S^{2}\right) \rightarrow H_{S^{1}}^{\bullet}(\{N\}) \approx k[u]$ sending $x \mapsto-u$ and $u \mapsto u$. We omit the details of computing the bar complex, but one finds without difficulty the cohomology generators $(1, \underbrace{x, \ldots, x}_{n}, 1)$ in degree $n$ for $n$ odd, and $\left(u^{n / 2}, 1\right)$ in degree $n$ for $n$ even. Owing to the shuffle product structure on the bar complex, one sees that, as an $R$-algebra,

$$
H_{S^{1}}^{\bullet}\left(\Omega S^{2}\right) \approx k[u] \oplus \bigwedge\left(x_{1}\right) \oplus \bigwedge\left(x_{3}\right) \oplus \bigwedge\left(x_{5}\right) \ldots
$$

where $x_{i}$ is an indeterminant of degree $i$.

Remark 7.1. In this example, the normalized bar complex $\bar{B}_{R}\left(R, H_{S^{1}}\left(S^{2}\right), R\right)$ is actually isomorphic to the $k$ CDGA minimal model for $E S^{1} \times{ }_{S^{1}} \Omega S^{2}$, which is

$$
\mathcal{M}_{E S^{1} \times{ }_{S} 1} \Omega S^{2}=\bigwedge(u, x, y) \quad(d u=0=d x, d y=u x)
$$


where the degrees of $u$ and $y$ are 2 , and the degree of $x$ is 1 . The isomorphism is given by $(1, x, 1) \mapsto x,(u, 1) \mapsto u$, and $(1, x, x, 1) \mapsto y$.

Remark 7.2. In this example, the space $E S^{1} \times{ }_{S^{1}} \Omega S^{2}$ is not formal, implying that $\Omega S^{2}$ is not $G$-formal. Indeed, Massey products abound.

\section{Appendix A. Bar complexes And Eilenberg-Moore theory}

In this appendix we shall discuss the theory of Eilenberg and Moore concerning pull-backs of fibrations. We will also consider equivariant versions of these results. For references, see [20, [24], or 8].

Let us suppose that we have a fibration $F \rightarrow E \stackrel{p}{\rightarrow} B$ and a map $f: X \rightarrow B$, so that we obtain a pull-back diagram:

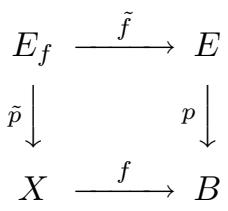

Then the maps $f^{*}$ and $p^{*}$ make $A^{\bullet}(X)$ and $A^{\bullet}(E)$ (differential graded) modules over $A^{\bullet}(B)$. Let us assume that $B$ is simply-connected. Then a theorem of Eilenberg and Moore asserts that there is an isomorphism

$$
\theta: \operatorname{Tor}_{A} \bullet(B)\left(A^{\bullet}(X), A^{\bullet}(E)\right) \rightarrow H^{\bullet}\left(E_{f}\right) .
$$

We may use the bar resolution to obtain a resolution of, say, $A^{\bullet}(X)$ by $A^{\bullet}(B)$ modules. Since we are considering $A^{\bullet}(-)$ to be the de Rham or Sullivan-de Rham complex, we will use Chen's normalized bar resolution, see [5] or [10].

More specifically, the bar complex is

$$
B_{k}\left(A^{\bullet}(X), A^{\bullet}(B), A^{\bullet}(E)\right)=\bigoplus_{i=0}^{\infty} A^{\bullet}(X) \otimes_{k}\left(s A^{\bullet}(B)\right)^{\otimes i} \otimes_{k} A^{\bullet}(E),
$$

where the tensor products are over the ground field $k$, and $s$ denotes the suspension functor on graded vector spaces that lowers the degree by one. Hence the degree of an element $\left(\alpha, \omega_{1}, \ldots, \omega_{k}, \beta\right)$ is $\operatorname{deg}(\alpha)+\sum_{i=1}^{k}\left(\operatorname{deg}\left(\omega_{i}\right)-1\right)+\operatorname{deg}(\beta)$, where $\alpha \in A^{\bullet}(X), \omega_{i} \in A^{\bullet}(B)$, and $\beta \in A^{\bullet}(E)$. Actually, the bar complex is bigraded. We introduce the bar degree, denoted $B_{k}\left(A^{\bullet}(X), A^{\bullet}(B), A^{\bullet}(E)\right)$.. The bar degree of an element $\left(\alpha, \omega_{1}, \ldots, \omega_{k}, \beta\right)$ is defined to be $-k$. The other grading is the normal tensor product grading, the degree of an element $\left(\alpha, \omega_{1}, \ldots, \omega_{k}, \beta\right)$ being $\operatorname{deg}(\alpha)+\sum_{i=1}^{k} \operatorname{deg}\left(\omega_{i}\right)+\operatorname{deg}(\beta)$. 
There are two differentials of total degree +1 :

$$
\begin{aligned}
d\left(\alpha, \omega_{1}, \ldots, \omega_{k}, \beta\right) & =\left(d \alpha, \omega_{1}, \ldots, \omega_{k}, \beta\right) \\
& +\sum_{i=1}^{k}(-1)^{\varepsilon_{i-1}+1}\left(\alpha, \omega_{1}, \ldots, \omega_{i-1}, d \omega_{i}, \omega_{i+1}, \ldots, \omega_{k}, \beta\right) \\
& +(-1)^{\varepsilon_{k}}\left(\alpha, \omega_{1}, \ldots, \omega_{k}, d \beta\right) \\
-\delta\left(\alpha, \omega_{1}, \ldots, \omega_{k}, n\right) & =(-1)^{\varepsilon_{0}}\left(\alpha \omega_{1}, \omega_{2}, \ldots, \omega_{k}, \beta\right) \\
& +\sum_{i=1}^{k-1}(-1)^{\varepsilon_{i}}\left(\alpha, \omega_{1}, \ldots, \omega_{i-1}, \omega_{i} \omega_{i+1}, \omega_{i+2}, \ldots, \omega_{k}, \beta\right) \\
& +(-1)^{\varepsilon_{k-1}+1}\left(\alpha, \omega_{1}, \ldots, \omega_{k-1}, \omega_{k} \beta\right),
\end{aligned}
$$

where $\varepsilon_{i}=\operatorname{deg} \alpha+\operatorname{deg} \omega_{1}+\cdots+\operatorname{deg} \omega_{i}-i$. The differential $\delta$ has degree +1 with respect to the bar grading, while the differential $d$ has degree +1 with respect to the tensor product grading. One may verify that $d \delta+\delta d=0$, and we put $D \stackrel{\text { def }}{=} d+\delta$ to be the total differential. With the given bigrading, we get a double complex with the two differentials $d$ and $\delta$, which gives rise to the Eilenberg-Moore spectral sequence.

Chen's normalized version of this bar complex is the following. If $f \in A^{0}(B)$, let $S_{i}(f)$ be the operator on $B\left(A^{\bullet}(X), A^{\bullet}(B), A^{\bullet}(E)\right)$ defined by

$$
S_{i}(f)\left(\alpha, \omega_{1}, \ldots, \omega_{k}, \beta\right)=\left(\alpha, \omega_{1}, \ldots, \omega_{i-1}, f, \omega_{i}, \ldots, \omega_{k}, \beta\right)
$$

for $1 \leq i \leq k+1$. Let $W$ be the subspace of $B\left(A^{\bullet}(X), A^{\bullet}(B), A^{\bullet}(E)\right)$ generated by the images of $S_{i}(f)$ and $D S_{i}(f)-S_{i}(f) D$. Then define

$$
\bar{B}\left(A^{\bullet}(X), A^{\bullet}(B), A^{\bullet}(E)\right) \stackrel{\text { def }}{=} B\left(A^{\bullet}(X), A^{\bullet}(B), A^{\bullet}(E)\right) / W .
$$

Then $W$ is closed under $D$, and when $H^{0}(B)=k$ ( $B$ is connected), then $W$ is acyclic, so that $\bar{B}\left(A^{\bullet}(X), A^{\bullet}(B), A^{\bullet}(E)\right)$ is quasi-isomorphic to $B\left(A^{\bullet}(X), A^{\bullet}(B)\right.$, $\left.A^{\bullet}(E)\right)$. Notice that in the normalized bar complex there are no elements of negative degree, and with our assumption that $B$ is simply-connected, we are assured convergence of the associated Eilenberg-Moore spectral sequence. The map $\theta$ mentioned above is induced by the map

$$
\theta: B\left(A^{\bullet}(X), A^{\bullet}(B), A^{\bullet}(E)\right) \rightarrow A^{\bullet}\left(E_{f}\right),
$$

which sends all tensor products to zero except for $A^{\bullet}(X) \otimes_{k} A^{\bullet}(E)$, where the map is $(\alpha, \beta) \mapsto \tilde{p}^{*} \alpha \wedge \tilde{f}^{*} \beta$. Note that $\theta(W)=0$, so that we get an induced map

$$
\theta: \bar{B}\left(A^{\bullet}(X), A^{\bullet}(B), A^{\bullet}(E)\right) \rightarrow A^{\bullet}\left(E_{f}\right) .
$$

The normalized bar complex may also be augmented. The augmentation, $\varepsilon$, maps all elements of positive total degree to zero. The elements of degree zero have the form $(f, g)$, where $f \in A^{0}(X)$ and $g \in A^{0}(E)$. Then we define $\varepsilon(f, g)=$ $\varepsilon_{X}(f) \varepsilon_{E}(g)=f\left(x_{0}\right) g\left(e_{0}\right)$, where $x_{0}$ and $e_{0}$ are chosen base points in $X$ and $E$, respectively, and $\varepsilon_{X}, \varepsilon_{E}$ are the augmentations of $A^{\bullet}(X), A^{\bullet}(E)$, respectively. If we choose base points so that the pull-back diagram above preserves all base points, then $\theta$ is an augmentation-preserving map.

The bar complex has a natural coalgebra structure. Since we are inputting $k$ CDGA's to the bar complex, we also obtain a structure of $k$ CDGA on the bar complex via the shuffle product. 
More specifically, if $\left(a_{1}, \ldots, a_{p}\right)$ and $\left(b_{1}, \ldots, b_{q}\right)$ are two ordered sets, then a shuffle $\sigma$ of $\left(a_{1}, \ldots, a_{p}\right)$ with $\left(b_{1}, \ldots, b_{q}\right)$ is a permutation of the ordered set $\left(a_{1}, \ldots, a_{p}\right.$, $\left.b_{1}, \ldots, b_{q}\right)$ that preserves the order of the $a_{i}$ 's as well as the order of the $b_{j}$ 's. That is, we demand that if $i<j$, then $\sigma\left(a_{i}\right)<\sigma\left(a_{j}\right)$ and $\sigma\left(b_{i}\right)<\sigma\left(b_{j}\right)$.

We obtain a product on $B\left(A^{\bullet}(X), A^{\bullet}(B), A^{\bullet}(E)\right)$ by first taking the normal tensor product on the $A^{\bullet}(X) \otimes A^{\bullet}(E)$ factors, then taking the tensor product of this product with the shuffle product on the $A^{\bullet}(B)^{\otimes i}$ factors. As usual, we introduce a sign $(-1)^{\operatorname{deg}(\alpha) \operatorname{deg}(\beta)}$ whenever $\alpha$ is moved past $\beta$. One checks that this product induces a product on Chen's normalized complex, $\bar{B}\left(A^{\bullet}(X), A^{\bullet}(B), A^{\bullet}(E)\right)$, as well. Thus we arrive at the following lemma, whose proof is left to the reader. For more details, see [16].

Lemma A.1. Assume that we have the pull-back diagram (46), where $p$ is a fibration and $B$ is simply-connected. Then the normalized bar complex

$$
\bar{B}\left(A^{\bullet}(X), A^{\bullet}(B), A^{\bullet}(E)\right)
$$

is a $k C D G A$. Moreover,

$$
\theta: \bar{B}\left(A^{\bullet}(X), A^{\bullet}(B), A^{\bullet}(E)\right) \rightarrow A^{\bullet}\left(E_{f}\right)
$$

is a quasi-isomorphism of $k C D G A$ 's.

Remark A.2. We note that Chen's normalization is functorial. That is, if we have a commutative diagram of $k$ CDGA's

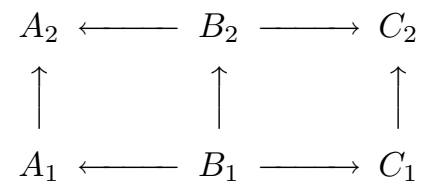

then we get a map of $k$ CDGA's $\bar{B}\left(A_{1}, B_{1}, C_{1}\right) \rightarrow \bar{B}\left(A_{2}, B_{2}, C_{2}\right)$.

We may formulate an equivariant analog of the bar complex. Let us consider again the pull-back diagram (46). If we suppose further that $X, B$, and $E$ are $G$ spaces, and that $f$ and $p$ are equivariant maps, then we obtain a pull-back diagram

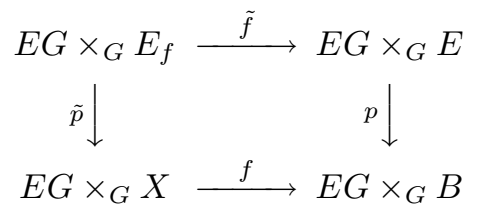

Note that we are assuming $B$ to be simply-connected, which in turn implies that $E G \times{ }_{G} B$ is simply-connected as well. We may apply Lemma A.1 to the diagram (56). However, the bar complex $\bar{B}\left(A_{G}^{\bullet}(X), A_{G}^{\bullet}(B), A_{G}^{\bullet}(E)\right)$ has the extra structure of an $R \mathrm{CDGA}^{\circ}$ or $R \mathrm{CDGA}$, depending on fixed points. We may give it an $R$ algebra strucure via the $R$-algebra structure on the $A_{G}^{\bullet}(X)$ factor, and we define the augmentation as above, assuming that we can choose our base points as described before to be actually fixed points of the group action. We arrive at the following.

Lemma A.3. Assume that in the pull-back diagram (46), we have that $X, B$, and $E$ are all $G$-spaces with $f$ and $p$ equivariant maps. Then the normalized bar complex

$$
\bar{B}\left(A_{G}^{\bullet}(X), A_{G}^{\bullet}(B), A_{G}^{\bullet}(E)\right)
$$


is an $R C D G A^{\circ}$. Moreover,

$$
\theta: \bar{B}\left(A_{G}^{\bullet}(X), A_{G}^{\bullet}(B), A_{G}^{\bullet}(E)\right) \rightarrow A_{G}^{\bullet}\left(E_{f}\right)
$$

is a quasi-isomorphism of $R C D G A^{o}$ 's. If we assume further that all fixed-point sets are non-empty, and the diagram (46) preserves base points chosen from the various fixed-point sets, then the normalized bar complex is an RCDGA, and $\theta$ is a quasi-isomorphism of RCDGA's.

In this equivariant case, we may further simplify the bar complex, following an idea of [10]. Let us consider the bar complex over $R$ :

$$
B_{R}\left(A_{G}^{\bullet}(X), A_{G}^{\bullet}(B), A_{G}^{\bullet}(E)\right)=\bigoplus_{i=0}^{\infty} A_{G}^{\bullet}(X) \otimes_{R}\left(s A_{G}^{\bullet}(B)\right)^{\otimes i} \otimes_{R} A_{G}^{\bullet}(E),
$$

where all the tensor products are over $R$.

Lemma A.4. Suppose that $A, B$, and $C$ are $R C D G A$ 's and we have morphisms of $R C D G A$ 's $A \leftarrow B \rightarrow C$, where $R=H^{\bullet}(B G)$ for $G$ a compact, connected Lie group. (We use this sequence to define a (differential graded) B-module structure on $A$ and $C$.) Suppose, further, either that for each $r \in R, r$ is not a zero-divisor in $A$, or that this condition holds for $C$. Then the natural projection

$$
B_{k}(A, B, C) \rightarrow B_{R}(A, B, C)
$$

is a quasi-isomorphism of RCDGA's.

Proof. We have that $B_{R}(A, B, C)=B_{k}(A, B, C) / V$, where $V$ is the sub-complex generated by all elements of the form

$$
\left(a, b_{1}, \ldots, r b_{i}, \ldots, b_{k}, c\right)-\left(a, b_{1}, \ldots, r b_{i+1}, \ldots, b_{k}, c\right)
$$

where $r \in R, a \in A, b_{j} \in B$, and $c \in C$. It is due to the fact that all elements of $R$ have even degree that $V$ is closed under the differential $D=d+\delta$. We claim that $V$ is, in fact, acyclic. To see this, consider the map $s: V^{i} \rightarrow V^{i-1}$ defined by

$$
\begin{aligned}
& s\left\{\left(a, b_{1}, \ldots, r b_{i}, \ldots, b_{k}, c\right)-\left(a, b_{1}, \ldots, r b_{i+1}, \ldots, b_{k}, c\right)\right\} \\
& =(-1)^{\varepsilon_{i}}\left\{\left(a, b_{1}, \ldots, r b_{i}, 1, b_{i+1}, \ldots, b_{k}, c\right)-\left(a, b_{1}, \ldots, b_{i}, r, b_{i+1}, \ldots, b_{k}, c\right)\right\}
\end{aligned}
$$

where $\varepsilon_{i}=\operatorname{deg} \alpha+\operatorname{deg} \omega_{1}+\cdots+\operatorname{deg} \omega_{i}-i$. It is straightforward but tedious to check that $d s+s d=0$, and that $\delta s+s \delta=i d$., so that $D s+s D=i d$., and consequently $V$ is acyclic. Moreover, it is easy to check that $V$ is an ideal, so that the product on the bar complex induces a product on the bar complex over $R$.

Remark A.5. Lemma A.4 is valid using the normalized bar complex.

Corollary A.6. In the situation of Lemma A.3,

$$
\theta: \bar{B}_{R}\left(A_{G}^{\bullet}(X), A_{G}^{\bullet}(B), A_{G}^{\bullet}(E)\right) \rightarrow A_{G}^{\bullet}\left(E_{f}\right)
$$

is a quasi-isomorphism of $R C D G A^{\circ}$ 's (RCDGA's). 


\section{REFERENCES}

1. C. Allday, Rational homotopy and torus actions, Houston Journal of Math. 5 (1979), 1-19. MR 80m:57033

2. - Invariant Sullivan-de Rham forms on cyclic sets, J. London Math. Soc. 57 (1998), 478-490. MR 99g:55015

3. C. Allday and V. Puppe, Cohomological methods in transformation groups, Cambridge University Press, 1993. MR 94g:55009

4. A. K. Bousfield and V.K.A.M. Gugenheim, On PL de Rham theory and rational homotopy type, Memoirs of the American Mathematical Society, no. 179, 1976. MR 54:13906

5. K. T. Chen, Reduced bar constructions on de Rham complexes, Algebra, Topology, and Category Theory, pp. 19-32, Academic Press, New York, 1976. MR 54:1272

6. P. Deligne, P. Griffiths, J. Morgan, and D. Sullivan, Real homotopy theory of Kähler manifolds, Invent. Math. 29 (1975), 245-275. MR 52:3584

7. W. G. Dwyer and J. Spalinski, Homotopy theories and model categories, Handbook of Algebraic Topology (I.M. James, ed.), Elsevier Science B.V., 1995, pp. 73-126. MR 96h:55014

8. S. Eilenberg and J. C. Moore, Homology and fibrations I, Coalgebras, cotensor product and its derived functors, Comment. Math. Helvetica 40 (1966), 199-236. MR 34:3579

9. A. Borel, Seminar on transformation groups, Ann. of Math. Studies, vol. 46, Princeton University Press, 1960. MR 22:7129

10. E. Getzler, J. D. S. Jones, and S. Petrack, Differential forms on loop spaces and the cyclic bar complex, Topology 30 (1991), 339-371. MR 92i:58179

11. M. Goresky, R. Kottwitz, and R. MacPherson, Equivariant cohomology, Koszul duality, and the localization theorem, Invent. Math. 131 (1998), 25-83. MR 99c:55009

12. P.-P. Grivel, Formes différentielles et suites spectrales, Ann. Inst. Fourier 29 (1979), 17-37. MR 81b:55041

13. V. Guillemin and S. Sternberg, Supersymmetry and equivariant de Rham theory, SpringerVerlag, New York, 1999. MR 2001i:53140

14. S. Halperin, Lectures on minimal models, Mém. Soc. Math. France, vols. 9-10, 1983. MR 85i:55009

15. S. Halperin and J. Stasheff, Obstructions to homotopy equivalences, Advances in Mathematics 32 (1979), 233-279. MR 80j:55016

16. S. Lillywhite, Bar complexes and formality of pull-backs, Preprint.

17. Maryland, College Park, MD., 1998.

18. space, Topology 39 (2000), 487-494. MR 2001b:55028

19. G. Lupton, Variations on a conjecture of Halperin, Homotopy and Geometry, vol. 45, Banach Center Publications, 1998, pp. 115-135. MR 99m:55014

20. J. McCleary, User's guide to spectral sequences, Publish or Perish, Inc., Wilmington, DE, 1985. MR 87f:55014

21. T. J. Miller, On the formality of $(k-1)$-connected compact manifolds of dimension less than or equal to $4 k-2$, Illinois Journal of Math. 23 (1979), 253-258. MR 80j:55017

22. D. G. Quillen, Homotopical algebra, Lecture Notes in Mathematics, vol. 43, Springer-Verlag, 1967. MR 36:6480

23. _ Rational homotopy theory, Ann. of Math. 90 (1969), 205-295. MR 41:2678

24. L. Smith, Homological algebra and the Eilenberg-Moore spectral sequence, Trans. Amer. Math. Soc. 129 (1967), 58-93. MR 35:7337

25. C. Teleman, The quantization conjecture revisited, Ann. of Math 152 (2000), 1-43. MR 2002d:14073

Department of Mathematics, University of Toronto, 100 St. George St., Toronto, Ontario, Canada M5S 3G3

E-mail address: sml@math.toronto.edu 